\title{
DÜBLIN
}

Technological University Dublin

ARROW@TU Dublin

2006-01-01

\section{Design of Integrated Polarization Beam Splitter with Liquid Crystal}

\author{
Qian Wang \\ Technological University Dublin \\ Gerald Farrell \\ Technological University Dublin, gerald.farrell@tudublin.ie \\ Yuliya Semenova \\ Technological University Dublin, yuliya.semenova@tudublin.ie
}

Follow this and additional works at: https://arrow.tudublin.ie/engscheceart

Part of the Electrical and Computer Engineering Commons

\section{Recommended Citation}

Wang, Q., Farrell, G., Semenova, Y.: Design of integrated polarization beam splitter with liquid crystal. IEEE Journal of Selected Topics in Quantum Electronics, Special issue devoted to Silicon Photonics, 2006, Vol.12, no.6 part 2, pp.1349-1353. doi:10.1109/JSTQE.2006.883156

This Article is brought to you for free and open access by the School of Electrical and Electronic Engineering at ARROW@TU Dublin. It has been accepted for inclusion in Articles by an authorized administrator of ARROW@TU Dublin. For more information, please contact arrow.admin@tudublin.ie, aisling.coyne@tudublin.ie, gerard.connolly@tudublin.ie.

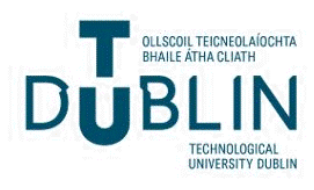




\title{
Design of Integrated Polarization Beam Splitter With Liquid Crystal
}

\author{
Qian Wang, Gerald Farrell, and Yuliya Semenova
}

\begin{abstract}
An integrated polarization beam splitter based on a directional coupler (DC) with an etched slot and filled-in liquid crystal covering layer, which utilizes the birefringence of liquid crystal materials, is proposed. A supermode solution for the DC involving birefringence materials and the corresponding full-vector anisotropic beam propagation method are employed as modeling tools. A numerical design of the proposed structure is presented. Beam propagation behavior along the whole structure involving curved input and output regions and central coupling region is simulated. Simulation result shows an extinction ratio of 25.3 and $41.9 \mathrm{~dB}$ for the TE and TM mode, respectively.
\end{abstract}

Index Terms-Directional coupler (DC), liquid crystals, polarization beam splitter (PBS).

\section{INTRODUCTION}

$\mathbf{P}$ OLARIZATION beam splitters (PBS), performing splitting or combining beams with orthogonal polarization states spatially, are widely used in optical communications or optical fiber sensing systems. Various waveguide-based integrated PBS have been proposed recently. Examples include asymmetric $Y$-branches [1], [2], directional couplers (DCs) with stressinduced birefringence [3], metal-clad Mach-Zehnder interferometers [4] or deeply etched multimode interference couplers (MMI) [5], etc. Some of these proposed structures require special fabrication procedures or exhibit a poor extinction ratio between the two outputs. The PBS based on the DC with stressinduced birefringence [3] has a simple configuration but suffers from the long coupling length because of the weak birefringence.

To shorten the length of the device, liquid crystal material was introduced in [6] because of its relatively large birefringence. However, the corresponding reported structure consists of two coupling waveguides on two different substrates, which increases the difficulty of assembly and coupling to fibers. Furthermore, the modeling and design in [6] is based on the twodimensional (2-D) scalar-wave equation; hence, the waveguide is treated as a slab waveguide. However, the liquid crystal material is a birefringence dielectric structure and, in practice, the waveguides have limited cross sections.

We first propose an improved configuration, in which the DC is integrated on one substrate. Secondly, we propose a supermode solution based on three-dimensional (3-D) quasivector wave equations and a 3-D full-vectorial beam propagation method (BPM) as modeling tools, which consider the birefringence dielectric structure of liquid crystals and limited cross

Manuscript received October 28, 2005; revised August 9, 2006.

The authors are with the Applied Optoelectronics Centre, School of Electronics and Communications Engineering, Dublin Institute of Technology, Dublin 8, Ireland (e-mail: qian.wang@dit.ie).

Digital Object Identifier 10.1109/JSTQE.2006.883156 section of waveguides. Using these two modeling tools, the proposed integrated polarization splitter is designed and simulated numerically in Section III. In the design procedure, the coupling in the curved input and output regions is also considered. The simulation result for the whole structure shows an extinction ratio of 25.3 and $41.9 \mathrm{~dB}$ for the TE and TM mode, respectively.

\section{Proposed Configuration AND Modeling ToOls}

\section{A. Proposed Configuration}

The schematic structure of the proposed configuration is presented in Fig. 1. A slot is etched in the middle of the DC. The liquid crystal is filled in and employed as a covering layer as well. The whole device can be regarded as a liquid crystal cell with planar lightwave circuits integrated on one substrate. There are two possible alignment directions for the liquid crystal molecules, which can be along the $x$ axis (parallel alignment; configuration A) or the $y$ axis (perpendicular alignment; configuration $\mathrm{B}$ ). Both configurations $\mathrm{A}$ and $\mathrm{B}$ involve the alignment of liquid crystal on surfaces of the top glass plate and the substrate as far as the fabrication of the device is concerned. For configuration $\mathrm{A}$, the planar alignment on the top glass surface can be realized by coating a polymer layer and rubbing with a nylon cloth similar to the method used in the manufacturing of liquid crystal displays. For the substrate, the liquid crystal can be aligned by evaporating a thin layer of silicon monoxide (about $20 \mathrm{~nm}$ ) on the substrate as experimentally demonstrated in [7]. For configuration B, the perpendicular alignment of the liquid crystal at surfaces of both the glass and the substrate can be implemented by treating the surface with a low-surface energy surfactant as shown in [8]. The ordinary and extraordinary refractive indices of the liquid crystals are $n_{o}$ and $n_{e}$, respectively (here, a liquid crystal of positive birefringence is chosen, i.e., $n_{e}>n_{o}$ ). The refractive index of the other cladding area $n_{\mathrm{cl}}$ is chosen to be equal to $n_{e}$ in this paper. The width and height of the etched slot is denoted as $w$ and $h$, respectively.

The operational mechanism of this polarization beam splitter is due to the birefringence of the liquid crystal. The TE and TM modes (polarization direction of the TE and TM mode is defined along $x$ axis and $y$ axis, respectively) have different index profiles in the liquid crystal area and consequently, have different propagation characteristics. In configuration A of Fig. 1, the TE mode is supposed to be coupled to the other waveguide at an appropriate length, but TM mode remains in the same waveguide within that length due to the strong guiding while propagating along this DC. In configuration B, the TM mode will be coupled to the other waveguide and the TE mode will remain in the same waveguide. Compared to the configuration proposed in [6], for the present configurations, there is no need to assemble the two 


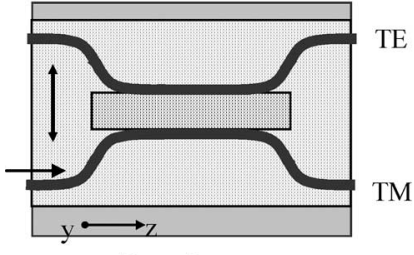

Top-view

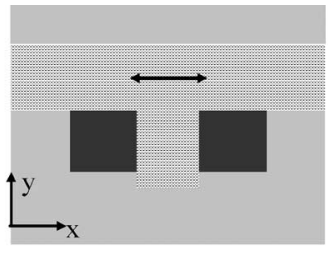

Cross-section
Configuration A

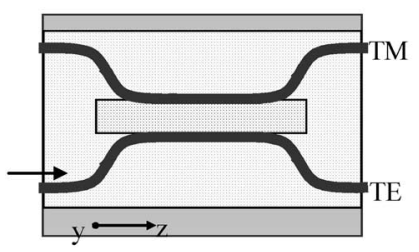

Top-view

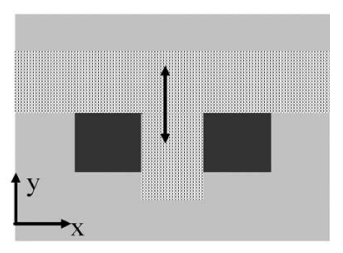

Cross-section
Configuration B

Fig. 1. Schematic structures of polarization splitter based on DC and filled-in liquid crystals; (LC) molecules are aligned along the $x$ axis in configuration A and along the $y$ axis in configuration B.

waveguides on two different substrates to make a DC, which imposes a very strict requirement to the fabrication tolerance. Since the DC is integrated on the same substrate in the present configuration, it is more convenient to couple with fibers than with the configuration in [6].

To carry out the design and simulation, supermode solution for the DC involving birefringence materials and corresponding full-vector BPM are briefly described.

\section{B. Modeling Tools}

The wave equation involving birefringence dielectric structure is

$$
\nabla \times \nabla \times \overrightarrow{\boldsymbol{E}}-k^{2} \hat{\epsilon}(x, y, z) \overrightarrow{\boldsymbol{E}}=0
$$

where $k=2 \pi / \lambda_{0}$ and $\lambda_{0}$ is wavelength in free space. In the liquid crystal area, $\hat{\varepsilon}$ is the optical tensor of liquid crystals. For configuration A: $\varepsilon_{x x}=n_{e}^{2}, \varepsilon_{y y}=\varepsilon_{z z}=n_{o}^{2}, \varepsilon_{m n}=0$ (where $m, n=x, y, z$, and $m \neq n$ ) and for configuration $\mathrm{B}: \varepsilon_{y y}=n_{e}^{2}, \varepsilon_{x x}=\varepsilon_{z z}=n_{o}^{2}, \varepsilon_{m n}=0$ (where $m, n=x, y, z$, and $m \neq n$ ). For both the configurations, in the core area $\varepsilon_{x x}=\varepsilon_{y y}=\varepsilon_{z z}=n_{c o}^{2}, \varepsilon_{m n}=0$ and in the other cladding region, $\varepsilon_{x x}=\varepsilon_{y y}=\varepsilon_{z z}=n_{c l}^{2}, \varepsilon_{m n}=0$. The $x$ and $y$ component of the electric field satisfies

$$
-\frac{\partial^{2}}{\partial z^{2}}\left[\begin{array}{l}
E_{x} \\
E_{y}
\end{array}\right]=\left[\begin{array}{ll}
P_{x x} & P_{x y} \\
P_{y x} & P_{y y}
\end{array}\right]\left[\begin{array}{c}
E_{x} \\
E_{y}
\end{array}\right]
$$

where

$$
\begin{aligned}
& P_{x x}=\frac{\partial}{\partial x}\left[\frac{1}{\varepsilon_{z z}} \frac{\partial}{\partial x}\left(\varepsilon_{x x}\right)\right]+\frac{\partial^{2}}{\partial y^{2}}+k^{2}\left[\left(\varepsilon_{x x}-n_{0}^{2}\right)\right] \\
& P_{x y}=\frac{\partial}{\partial x}\left[-\frac{\partial}{\partial y}+\frac{1}{\varepsilon_{z z}} \frac{\partial}{\partial y}\left(\varepsilon_{y y}\right)\right] \\
& P_{y x}=\frac{\partial}{\partial y}\left[-\frac{\partial}{\partial x}+\frac{1}{\varepsilon_{z z}} \frac{\partial}{\partial x}\left(\varepsilon_{x x}\right)\right] \\
& P_{y y}=\frac{\partial^{2}}{\partial x^{2}}+\frac{\partial}{\partial y}\left[\frac{1}{\varepsilon_{z z}} \frac{\partial}{\partial y}\left(\varepsilon_{y y}\right)\right]+k^{2}\left[\left(\varepsilon_{y y}-n_{0}^{2}\right)\right] .
\end{aligned}
$$

By neglecting the coupling between TE and TM modes in the above equation, quasi-vector mode equation is obtained. For the DC, assume that the two supermodes for the quasi-TE and quasiTM mode have propagation constants $\beta_{i}^{\mathrm{TE}}$ and $\beta_{i}^{\mathrm{TM}}(i=1$, symmetric supermode; $i=2$, asymmetric supermode). Electric field components $E_{x}(x, y)$ and $E_{y}(x, y)$ of the quasi-vector mode satisfy the following equations, respectively,

$$
\begin{aligned}
& \frac{\partial}{\partial x}\left[\frac{1}{\varepsilon_{z z}} \frac{\partial}{\partial x}\left(\varepsilon_{x x} E_{x}\right)\right]+\frac{\partial^{2} E_{x}}{\partial y^{2}}+\left(k^{2} \varepsilon_{x x}-\left(\beta_{i}^{\mathrm{TE}}\right)^{2}\right) E_{x}=0 \\
& \frac{\partial^{2} E_{y}}{\partial x^{2}}+\frac{\partial}{\partial y}\left[\frac{1}{\varepsilon_{z z}} \frac{\partial}{\partial y}\left(\varepsilon_{y y} E_{y}\right)\right]+\left(k^{2} \varepsilon_{y y}-\left(\beta_{i}^{\mathrm{TM}}\right)^{2}\right) E_{y}=0 .
\end{aligned}
$$

The coupling length (the light is totally coupled from one waveguide to the other) of the DC can be calculated as $L_{c}^{\mathrm{TE}}=$ $\pi /\left(\beta_{1}^{\mathrm{TE}}-\beta_{2}^{\mathrm{TE}}\right)$ and $L_{c}^{\mathrm{TM}}=\pi\left(\beta_{1}^{\mathrm{TM}}-\beta_{2}^{\mathrm{TM}}\right)$, for $\mathrm{TE}$ ad $\mathrm{TM}$ modes, respectively. For a specific length of the DC $L$, the coupling ratios of the DC for TE and TM mode are, respectively, [9]

$$
R_{\mathrm{TE}}=\left|\frac{1-\cos \left(\left(\beta_{1}^{\mathrm{TE}}-\beta_{2}^{\mathrm{TE}}\right) L\right)}{1+\cos \left(\left(\beta_{1}^{\mathrm{TE}}-\beta_{2}^{\mathrm{TE}}\right) L\right)}\right|
$$

and

$$
R_{\mathrm{TM}}=\left|\frac{1-\cos \left(\left(\beta_{1}^{\mathrm{TM}}-\beta_{2}^{\mathrm{TM}}\right) L\right)}{1+\cos \left(\left(\beta_{1}^{\mathrm{TM}}-\beta_{2}^{\mathrm{TM}}\right) L\right)}\right| .
$$

Besides the above quasi-vector supermode solution, a fullvector BPM is employed to simulate beam behaviors for the designed structure, of which the two coupling items are considered. With the slowly varying envelope approximation $\left[E_{i}=\hat{E}_{i} \exp \left(j k n_{0} z\right),(i=x, y)\right]$ and paraxial assumption, they have the form

$$
-2 j k n_{0} \frac{\partial}{\partial z}\left(\left[\begin{array}{l}
\hat{E}_{x} \\
\hat{E}_{y}
\end{array}\right]\right)=\left[\begin{array}{ll}
P_{x x} & P_{x y} \\
P_{y x} & P_{y y}
\end{array}\right]\left[\begin{array}{l}
\hat{E}_{x} \\
\hat{E}_{y}
\end{array}\right] .
$$

These coupled equations can be solved by adopting an alternative direction implicit scheme as in [10], with which these equations are converted into 2-D beam propagation problems. The transparent condition or perfect matched layer can be used at the edges of the calculation region [11], [12].

The design and simulation of this integrated PBS obtained using these modeling tools are presented in the following section.

\section{NUMERICAL DESIGN AND SIMULATION}

Configuration $\mathrm{A}$ is considered as the numerical example for the design and analysis of the integrated polarization splitter (for configuration B the procedure is the same). The wavelength of the propagation light is chosen to be $1550 \mathrm{~nm}$, and the silica waveguide is considered because of its low propagation loss and high coupling efficiency to fibers. The ordinary and extraordinary refractive indices of the liquid crystal are $n_{o}=1.455$ and $n_{e}=1.505$, respectively. The cross section of the waveguide core is $5 \times 5 \mu \mathrm{m}^{2}$. The refractive index of the cladding region is $n_{\mathrm{cl}}=1.505$ and that of the core is $n_{\mathrm{co}}=1.515$. For the 


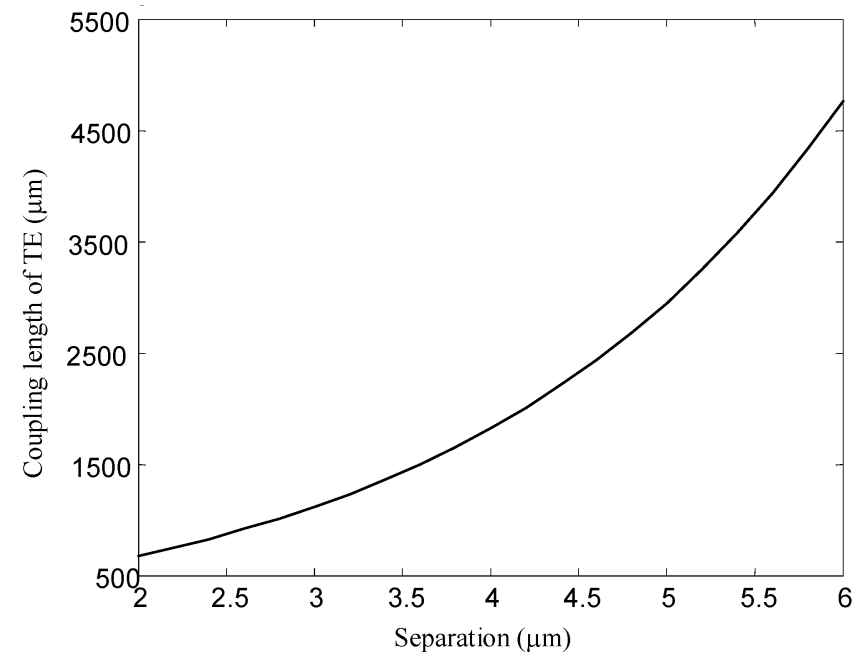

(a)

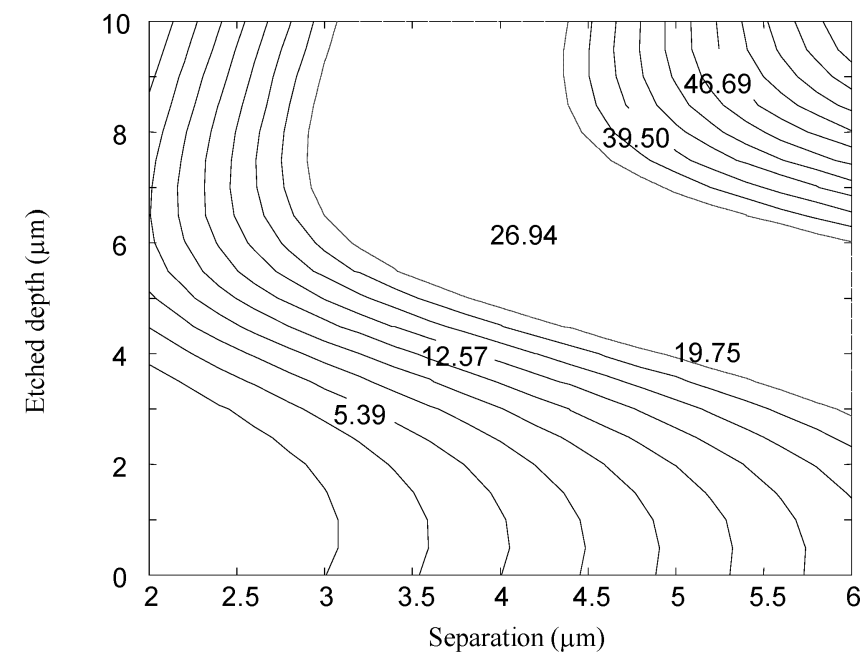

(b)

Fig. 2. (a) Coupling length of TE mode versus separation between two waveguides. (b) Contour plot of extinction ratio for the TM mode under different etched depth and separation using the corresponding coupling length of the TE mode as shown in (a).

TE mode, the coupling length varies as the separation of the two waveguides changes but remains almost the same for different etching depth of the slot. Fig. 2(a) gives the calculated coupling length of the TE mode while the separation varies from 2 to $6 \mu \mathrm{m}$. The extinction ratio of the TM mode is calculated for different etched depths and separations using the corresponding coupling length for the TE mode presented in Fig. 2(a) [see Fig. 2(b)].

As shown in Fig. 2(a), when the separation increases, the coupling length increases significantly. The coupling length for the TE mode is strongly dependent on the separation of the two waveguides; thus, when the separation increases, the coupling length increases significantly. The calculated results of Fig. 2(b) show that for a given separation, i.e., a given coupling length for the TE mode, the extinction ratio is increased as the etched depth increases (however, a deep etching increases the fabrication complexity and tolerance requirement) or for a given etched

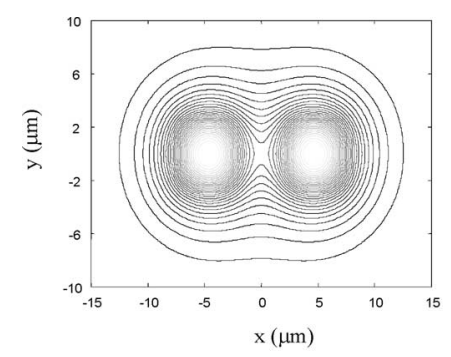

(a)

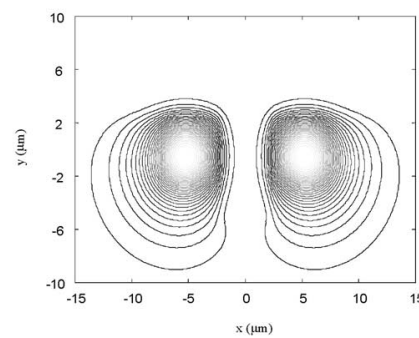

(c)

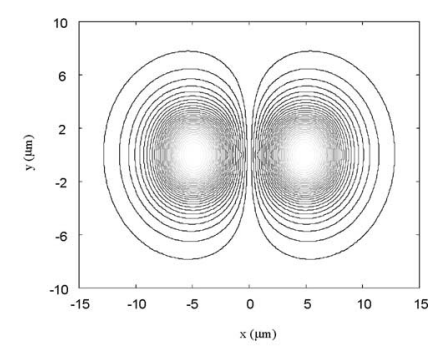

(b)

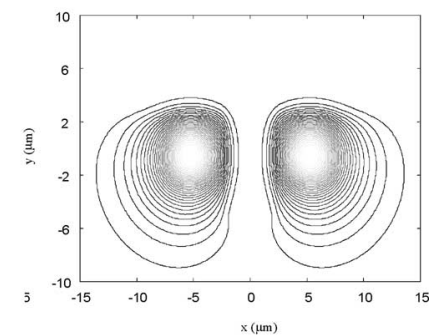

(d)
Fig. 3. Field distribution of the two supermodes for the DC. (a) Symmetric supermode of TE. (b) Asymmetric supermode of TE. (c) Symmetric supermode of TM. (d) Asymmetric supermode of TM.

depth it is also increased as the separation increases. The selection of the correct parameters of separation from Fig. 2(a) and (b) involves a compromise where achieving a compact size will restrict the extinction ratio. Conversely, selecting a high extinction results in a long coupling length for the TE mode. In this example, a separation of $4.5 \mu \mathrm{m}$ and an etched depth of $8 \mu \mathrm{m}$ are chosen. The corresponding coupling length for TE mode is 2200 $\mu \mathrm{m}$, which is calculated by the formula $L_{c}^{\mathrm{TE}}=\pi /\left(\beta_{1}^{\mathrm{TE}}-\beta_{2}^{\mathrm{TE}}\right)$, i.e., within this length the propagation light of the TE mode is completely coupled from one waveguide to the other waveguide, and the extinction ratio for the TM mode is $36 \mathrm{~dB}$ with the supermode solution. To further reduce the length of the device, a liquid crystal material with a higher birefringence can be used. It should be understood that for the same waveguide parameters and same extraordinary refractive index of the liquid crystal material, when reducing the value of the ordinary refractive index of liquid crystal material (i.e., increasing the birefringence), the TM mode has a higher extinction ratio due to the stronger guiding as the refractive index of the cladding between the two waveguides decreases. Therefore, one can choose a smaller separation, which leads to a smaller coupling length required for the TE mode while maintaining a high extinction ratio for the TM mode.

When the waveguide separation is $4.5 \mu \mathrm{m}$ and the etched depth is $8 \mu \mathrm{m}$, the effective refractive indices of the two supermodes for the TE mode are 1.5092257 and 1.5088733 . Corresponding field distributions are shown in Fig. 3(a) and (b), respectively. For the TM mode, it has a different index profile because of the birefringence of liquid crystal. The effective refractive indices for the two supermodes are 1.5073871 and 1.5073845. Fig. 3(c) and (d) presents the corresponding field distributions. Fig. 3(c) and (d) indicates that the light fields of the supermodes are well defined for the two waveguides, which 


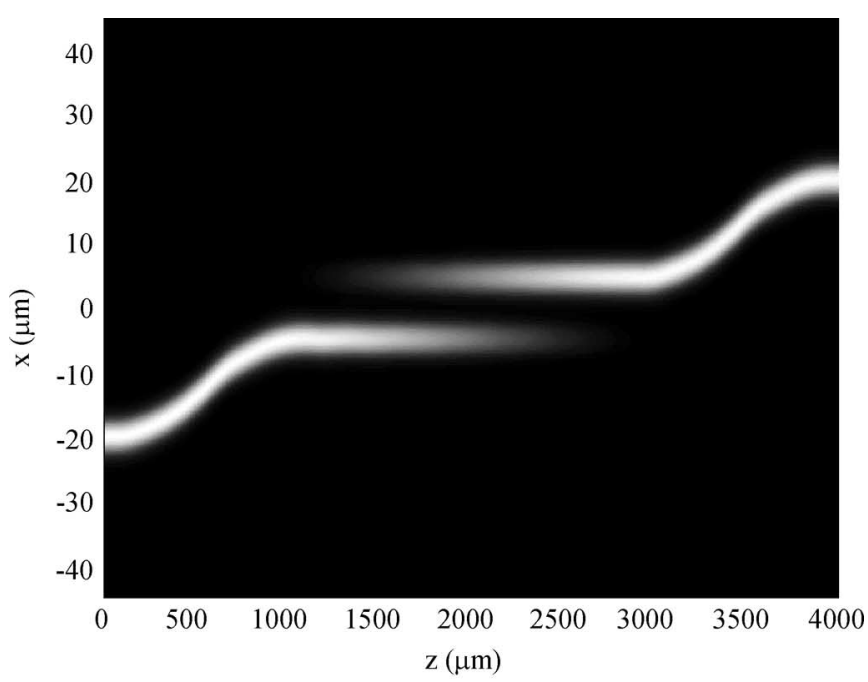

(a)

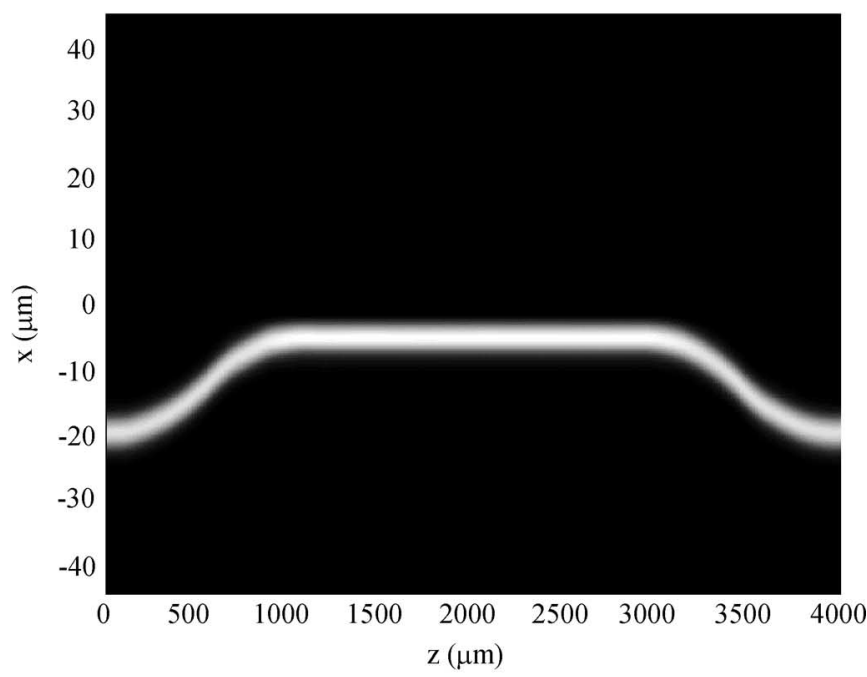

(b)

Fig. 4. Light intensity distribution while propagating along the whole structure based on the 3-D full-vector beam propagation method. (a) TE mode. (b) TM mode.

is significantly different from that shown in Fig. 3(a) and (b). The effective refractive indices of the two supermodes are very close, and consequently, the corresponding coupling length is very long. In summary, the coupling for the case of the TM mode between the two waveguides is very weak.

To split or combine the TE and TM modes in practice, the DC consists of curved input and output waveguides as shown in Fig. 1. It is known that due to coupling effect in the curved input and output region, the actual coupling length of the straight waveguide is shorter than the above presented designed length. With the effective design method proposed in [13], the actual length of the central region for the DC is $1787 \mu \mathrm{m}$ (for the curved input and output regions the bending radius $R$ is $20000 \mu \mathrm{m}$ and the separation is $40 \mu \mathrm{m})$.

Finally, the 3-D full-vector BPM is employed to simulate the light propagation along the whole structure, including the curved input region, the central coupling region, and the curved output region. Fig. 4 shows the light intensity distributions for the TE mode and the TM mode while propagating along the whole structure. Simulation results indicate that the TE mode is coupled to the other waveguide, while the TM mode remains in the same waveguide. The corresponding extinction ratio based on the simulation of the whole structure is 25.3 and $41.9 \mathrm{~dB}$ for the TE and TM mode, respectively.

\section{CONCLUSION}

A new integrated polarization beam splitter based on a DC with an etched slot and filled-in liquid crystal covering layer has been proposed in this paper. The birefringence of liquid crystal materials is used to split the TE and TM modes. Supermode solution for the DC involving birefringence materials and corresponding full-vector anisotropic BPM have been presented and employed as modeling tools for the design. Numerical design and simulation of the proposed structure has been demonstrated. Corresponding numerical result indicates an extinction ratio of 25.3 and $41.9 \mathrm{~dB}$ for the TE and TM mode, respectively. Compared to previous liquid crystal integrated polarization splitter, the proposed structure eases the difficulty of assembly and coupling to optical fibers due to integration of the two waveguides of the DC on the same substrate.

\section{REFERENCES}

[1] P. K. Wei and W. S Wang, "A TE-TM mode splitter on lithium niobate using Ti, Ni, and MgO diffusions," IEEE Photon. Technol. Lett., vol. 6, no. 2, pp. 245-248, Feb. 1994.

[2] S. M. Garner, V. Chuyanov, S. S. Lee, C. Antao, W. H. Steier, and L. R. Dalton, "Vertically integrated waveguide polarization splitters using polymers," IEEE Photon. Technol., Lett., vol. 11, no. 7, pp. 842-844, Jul. 1999.

[3] N. Miliou, R. Srivastava, and R. V. Ramaswamy, "A 1.3- $\mu$ m directional coupler polarization splitter by ion exchange," J. Lightw. Technol., vol. 11, no. 2, pp. 220-225, Feb. 1993.

[4] L. B. Soldano, A. I. de Vreede, M. K. Smit, B. H. Verbeek, E. G. Metaal, and F. H. Green, "Mach-Zehnder interferometer polarization splitter in InGaAsP/InP," IEEE Photon. Technol. Lett., vol. 6, no. 3, pp. 402-405, Mar. 1994.

[5] B. M. A. Rahman, N. Somasiri, C. Themistos, and K. T. V. Grattan, "Design of optical polarization splitters in a single-section deeply etched MMI waveguide," Appl. Phys. B, Photophys. Laser Chem., vol. 73, pp. 613618, 2001.

[6] K. C. Lin, W. C. Chuang, and W. Y. Lee, "Proposal and analysis of an ultrashort directional coupler polarization splitter with an NLC coupling layer," J. Lightw. Technol., vol. 14, no. 11, pp. 2547-2553, Nov. 1996.

[7] A. Zhang, K. T. Chan, M. S. Demokan, V. W. C. Chan, P. C. H. Chan, H. S. Kwok, and A. H. P. Chan, "Integrated liquid crystal optical switch based on total internal reflection," Appl. Phys. Lett., vol. 86, p. 211108, (1-3), 2005.

[8] G. P. Bryan-Brown, C. V. Brown, I. C. Sage, and V. C. Hui, "Voltagedependent anchoring of a nematic liquid crystal on a grating surface," Nature, vol. 392, pp. 365-367, 1998.

[9] F. Ladoucer and J. D. Love, Silica-Based Buried Channel Waveguides and Devices. $\quad$ London, U.K.: Chapman and Hall, 1996, pp. 145-158.

[10] Y. L. Hsueh, M. C. Yang, and H. C. Chang, "Three-dimensional noniterative full-vectorial beam propagation method based on the alternating direction implicit method," J. Lightw. Technol., vol. 17, no. 11, pp. 2389 2397, Nov. 1999.

[11] G. R. Hadley, "Transparent boundary condition for beam propagation," IEEE J. Quantum Electron., vol. 28, no. 1, pp. 363-370, Jan. 1992.

[12] W. P. Huang, C. L. Xu, W. Lui, and K. Yokoyama, "The perfectly matched layer (PML) for the beam propagation method," IEEE Photon. Technol. Lett., vol. 8, no. 5, pp. 649-651, May 1996.

[13] Q. Wang, S. He, and F. Chen, "An effective and accurate method for the design of directional couplers," IEEE J. Sel. Topics Quantum Electron., vol. 8, no. 6, pp. 1233-1238, Nov./Dec. 2002. 


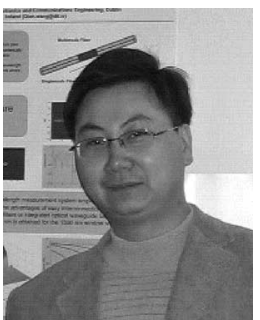

Qian Wang was born in China, in 1977. He received the Ph.D. degree in optical engineering from Zhejiang University, Zhejiang, China, in 2004.

In 2004, he joined the Applied Optoelectronics Centre, Dublin Institute of Technology, Dublin, Ireland, as a Postdoctoral Researcher. His current research interests include integrated optics, fiber optics, liquid crystals, optical communication and optical sensing, and automatic design of optics. He is the author of more than 40 published papers in these areas.

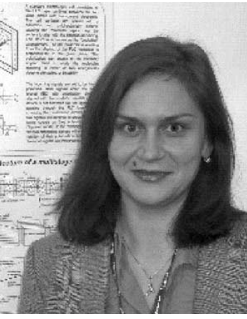

in the area of liquid crystals.
Yuliya Semenova received the B.E. degree in electronic engineering from the Lviv Polytechnic National University, Lviv, Ukraine, in 1992, and received the M.Sc. and the Ph.D. degrees in physics of liquid crystals.

She is currently a Postdoctoral Researcher and a part-time Lecturer in the Dublin Institute of Technology, Dublin, Ireland. She has carried out research, design, and teaching in the area of electronics, physics of liquid crystals, and their applications. She is the author of a number of journal and conference papers

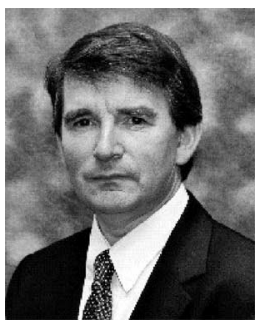

Gerald Farrell received the B.E. and M.E. degrees from University College Dublin, Dublin, Ireland, and Trinity College Dublin (TCD), Dublin, Ireland, in 1979 and 1983, respectively, and the Ph.D. degree in photonics from TCD, in 1993.

In 1995, he founded the Applied Optoelectronics Centre at Dublin Institute of Technology (DIT), Dublin, Ireland. Since 2001, he has been the Head of the School of Electronics and Communications Engineering in DIT. His current research interests include optical power and wavelength measurement, optical switching, plastic optical fibers and self-pulsation in laser diodes. He is the author of a considerable number of journal and conference papers in these areas. 